\title{
ON THE PATH OF A QUASI-STATIC CRACK IN MODE III
}

\author{
GERARDO E. OLEAGA
}

\begin{abstract}
A method for finding the path of a quasi-static crack growing in a brittle body is presented. The propagation process is modelled by a sequence of discrete steps optimizing the elastic energy released. An explicit relationship between the optimal growing direction and the parameters defining the local elastic field around the tip is obtained for an anti-plane field. This allows to describe a simple algorithm to compute the crack path.
\end{abstract}

\section{INTRODUCTION}

One of the basic problems in Fracture Mechanics consists in the prediction of crack paths once the loading and the initial configuration are given. The first elementary question that arises is how to quantify the deviation of the crack path from its plane with respect to the initial elastic field around the tip. We can divide this problem essentially in two steps: (a) to select a basic physical law for quasi-static propagation and (b) to compute an explicit relationship between "crack deviation" and "initial field" under the principle assumed in (a). In this article, we consider both problems for a crack growing in a quasi-static regime under the linear elastic theory for a Mode III field (anti-plane displacement). Our main results are the proposal and discussion of a discrete version of the Maximum Energy Release Rate criterion and an explicit formula linking the preferred direction of propagation in terms of the parameters defining the local field around the tip (cf. (4.17)). This relationship allows to describe a simple algorithm for the crack path as a sequence of straight segments. Before stating our approach it is worth to describe some of the relevant previous work done in connection to both problems (a) and (b).

In a two dimensional setting for brittle fracture we need at least two scalar equations to find the motion of the crack tip. One of them represents the energy conservation and is given by the Griffith condition:

$$
G:=-\lim _{l \rightarrow 0^{+}} \frac{\Delta E}{l}=\kappa .
$$

Here $\Delta E=E(l)-E(0)$ is the change of total mechanical energy in the body (elastic plus potential of the loading system) obtained while the crack performs an extension of length $l$ and $\kappa$ is a constant of the material called the surface energy. The quantity $G$ defined in (1.1) is the so-called Energy Release Rate. Equation (1.1) states that the body should release, per unit length, the same amount of mechanical energy as the one needed to separate the crack faces. In ([13]), Irwin showed that $G$ is a local quantity related to the stress intensity factors. If $G$ happens to be strictly lower than $\kappa$ there is no possible propagation: opening a small extension of the crack costs more than the energy available around the tip. On the other hand, if $G$ is strictly bigger than $\kappa$ there is an excess of stored energy which should be dissipated

Date: May 13, 2004

2000 Mathematics Subject Classification. 74R05, 74B05, 74G70.

Key words and phrases. Crack propagation, discrete model, singular fields, linear elasticity.

Supported by EU-Project "Front Singularities" University of Leipzig and the Max Planck Institute MIS. Partial support was also provided by the Spanish DGES project BFM2000-0605. 
through elastic waves (time dependent fields) or other dissipative phenomena. In the last case the propagation process is no more quasi-static.

Several criteria were introduced to provide the remaining equation. For the inplane modes of propagation, one of the most popular is the so-called symmetry principle (cf. [11]) which is written as:

$$
K_{\mathrm{II}}=0,
$$

where $K_{\mathrm{II}}$ is the stress intensity factor for Mode II. As stated by Cotterell and Rice in [6], this is a necessary condition if the crack path is assumed to be smooth. These authors studied (1.2) for paths slightly deviating from straightness and kinks, when it is applied to the tip far from a corner. It is to be noted that this principle cannot be applied in a pure Mode III setting and it is not evident how to generalize its formulation.

Another point of view is provided by the so-called Maximum Energy Release Rate criterion, which can be summarized as follows: given some crack configuration, the growing direction is selected in such a way that $G$ takes its maximum value. This provides a variational condition over the admissible paths and makes possible, at first sight, to detect the optimal growing strategy. As we will see later (see Section 4), this condition gives no information about the shape of the crack in a pure Mode III setting. It only tells us that the best way to grow is to follow the same direction as the one given by the actual configuration. This implies that abrupt changes in the direction of propagation (branches) are forbidden in an out-of-plane field.

A different approach, valid for all field modes, was followed recently by some authors (see [4], [9]). The fracture process is modelled as a discrete evolution of fairly general sets. At each step the crack configuration is found by minimizing the total energy of the crack-body system among the admissible competitors which are constrained to contain the path defined in the previous step of the process. We must emphasize that, in spite of the nice mathematical properties of this model (cf. [7], [8]), it predicts crack paths that may not be physically admissible according to Griffith's energy condition (1.1). Let us elaborate a bit on this point.

The total energy of the crack-body system is given by $E(l)+\kappa l$, where $E(l)$ is the mechanical energy (elastic + loading) and $\kappa l$ is the surface energy. In a global minimization step "big cracks" may appear and the details of the growing process are missed. To illustrate the situation we consider the following Figure. In this onedimensional setting, the model predicts that the crack will grow from position $l$ to position $l_{g l o b}$ through an interval where the the total energy is increasing. Therefore, $\frac{d E}{d l}+\kappa>0$ holds, and then $G=-\frac{d E}{d l}$ is strictly lower than $\kappa$, contradicting Griffith's condition (1.1). As we mentioned above, there is not enough "driving force" to push the tip in that interval and the crack is not allowed to grow beyond $l_{\text {loc }}$. By the same argument, in the interval where the total energy is decreasing we must have that $G>\kappa$. If $G-\kappa$ turns to be large the propagation process is no more quasistatic and other inertial or dissipative effects should be included in the model. It is fair to say that, excepting a limited class of loading conditions, the global energy minimizing steps may distort completely the crack shape, allowing inadmissible configurations. Some attempts to deal with the shortcomings of this model are presented by M. Buliga in [5].

Another non-physical consequence of the global-minimizer approach is the possible appearance of cracks in a region free of singularities (crack initiation). In 


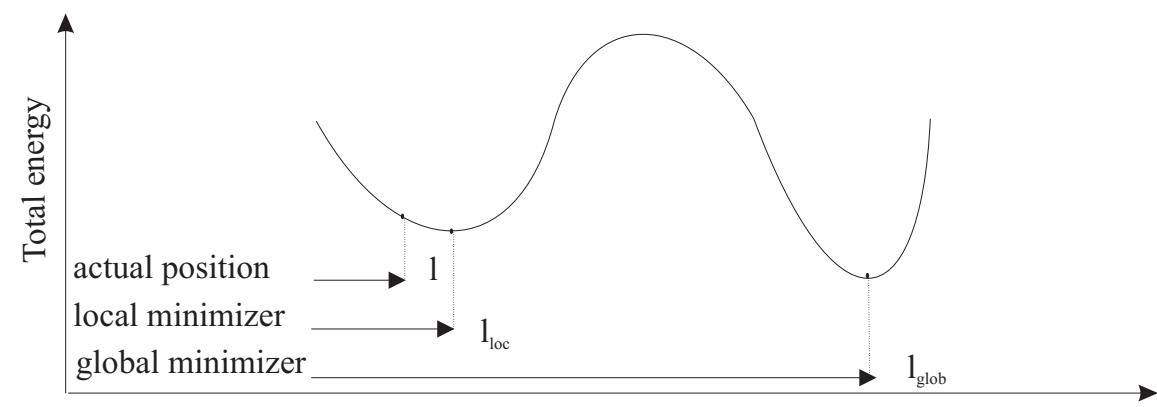

crack extension length

FiguRE 1. Global minimization

Grifftith's pioneering work [12], it is shown that for a straight cut the energy release rate $G$ at one of the tips is proportional to its length. Cracks of a small size will never have enough driving force at the tips to reach the threshold $\kappa$. Nevertheless, under a global energy minimizing process some cracks may appear in a "healthy" place: it could be more convenient, in terms of the total energy cost, to generate a finite cut even if this crack violates condition (1.1) during the propagation.

Our work is very close in spirit to the ones of Leblond [14], and Amestoy \& Leblond [1]. It is worth to mention here the similarities and to point out the differences between their approach and our setting. The main purpose of Leblond in [14] is to find the path of a crack in quasi-static propagation for the in-plane (modes I and II) displacement fields. To this end, he gives an expansion of the stress intensity factors in a general setting. In the second article ([1]) these authors provide the detailed form of the functions involved and they apply the results to study the symmetry principle. They perform a comparison between this principle and the maximum energy release rate criterion as well.

In this article we are also interested in the crack path, but for the case of a pure Mode III. In spite of the fact that the field equation is simpler, we must face two additional difficulties. As we mentioned above, it is not possible to apply the symmetry principle or the maximum energy release rate criterion, and we have to propose and discuss a natural way to formulate the propagation process from basic physical principles. The second difficulty is that we need a more detailed expansion of the field around the tip. In [1] it is shown that the stress intensity factors $K_{\mathrm{I}}$ and $K_{\mathrm{II}}$ (ie., the singular terms in the stress expansion) determine the main contribution to the deviation of a crack. In Mode III we show that it does not suffice to consider the value of $K_{\mathrm{III}}$, it is the next term in the field expansion (defined by $c_{2}$ in our setting, cf. (3.27)) the one that defines the shape of the growing crack (cf. formula (4.18)). We must then analyze the dependence of this term on crack extension together with the evolution of the stress intensity factor (cf. (3.36)). The last difference that we would like to mention is the fact that shapes of the form $y=a x^{3 / 2}$ are proposed in [14] in an ad-hoc manner while here, we obtain this paths as a consequence of the propagation law proposed.

The paper is organized as follows. We begin our discussion about the crack propagation criterion by revising the concept of Energy Release Rate in Section 
2. We describe a natural way to find the "optimal" growing direction using the spirit of Griffith's ideas. To be fair, this can be considered as a discrete version of the so-called maximum energy release rate criterion. We understand that all the physics of the growing process is concentrated at the tip of the crack and there is no global optimizer, global selection function or other simplified strategy for the crack path but successive instabilities near equilibrium configurations.

Sections 3 and 4 deal with the more technical aspects. In Section 3 we describe some basic facts about Mode III fields, as well as other relationships that are useful for later purposes. In Section 4 we find a fundamental relationship between the optimal crack growing direction and the coefficients of the local elastic field. We show that two terms in the asymptotic expansion of the energy released in terms of the crack length are needed in Mode III, while it is known that only the first one (ie., the energy release rate) is needed for the in-plane modes. The techniques applied are classical, including complex representation of the fields, Schwarz-Christoffel conformal mapping (cf. [15]) and elementary application of perturbation methods for algebraic equations (cf. [2]). We describe the discrete and quasi-static evolution of the path using the previous results, providing an algorithm of propagation. We conclude in Section 5 with some open questions. A simple derivation of the conformal transform applied is given in the Appendix.

\section{OUR BASIC APPROACH}

2.1. The Energy-Release-Rate concept revised. We begin by studying the quantity $G$ defined in (1.1) in more detail. It is known that its value depends on the overall geometry of the body-crack and the loading conditions, but it is sometimes bypassed that it depends on the trial paths as well. In other words, instead of using a straight virtual extension as the one used originally by Griffith in [12], we can add at the tip an arbitrary piece of curve $\gamma$ with a well defined arc-length parameter $l$ and consider $G$ as a functional depending on $\gamma$ as follows:

$$
G(\gamma):=-\lim _{l \rightarrow 0^{+}} \frac{\Delta E(l ; \gamma)}{l}
$$

where $E(l ; \gamma)$ defines the value of mechanical energy when the initial crack grows along the path $\gamma$ an extension $l$. We omit explicit reference to the loading and initial configuration in the notation.

Now we turn to the following question. If the value of $G(\gamma)$ depends on the trial extension, we may have some "unstable" paths for which $G$ is greater than $\kappa$, and some others that cannot be followed just because the energy to be released is less than the amount needed. A more precise statement should say that the configuration is in equilibrium if

$$
G(\gamma) \leq \kappa
$$

for all admissible extensions $\gamma$ of the crack. A very simple consequence of this formulation is the following: If all the paths $\gamma$ satisfying $G(\gamma)=\kappa$, are tangent to the same direction, then this will determine the unique way that the crack can follow inside this family. The critical path (or paths) $\gamma^{*}$ must therefore satisfy the following additional condition:

$$
G\left(\gamma^{*}\right)=\max _{\gamma} G(\gamma)
$$


This simple argument allows for studying the crack growth direction without further physical assumptions or any other ad-hoc propagation law. In a different context, (2.3) is referred to as the maximum energy release rate condition.

In the case of Modes I and II, the kinking angle could be in principle computed by applying condition (2.3). For smooth paths, some problems regarding crack shape were considered recently by M. Brokate and A. Khludnev in [3]. They studied the sensitivity of $G$ with respect to the subsequent crack path. In the case of Mode III it is possible to show that the best direction of propagation is always the same as the one given by the departing configuration, ie., the "best" kinking angle is always zero, as it can be seen in explicit formulae for $G$ (cf. for example [19]). Roughly speaking, any smooth path is compatible with (2.3) and we need to include more detailed information in the expansion for the energy released in terms of $l$.

2.2. A discrete approach. In order to find the main contribution of the elastic field to the direction of propagation, we allow the crack to perform a very small "jump" or fluctuation from its equilibrium position. This allows to consider the finite increment of the energy released and to obtain information using a more detailed expansion. Each jump will add a path of small length $l$ to the given configuration. Consider the finite increment:

$$
-\Delta E(l ; \gamma)=-(E(l ; \gamma)-E(0)) .
$$

Our aim is to estimate the optimal $\gamma^{*}$ satisfying:

$$
-\Delta E\left(l ; \gamma^{*}\right)=\max _{\gamma}(-\Delta E(l ; \gamma)),
$$

for $l>0$ small and fixed.

A simple way to quantify this point of view is to consider an indexed family of paths covering a wide variety of behaviors. In our setting, we take the kinked configurations with kinking angle $\varphi,|\varphi| \leq \pi$, indexed by the parameter $\alpha:=$ $\varphi / \pi$ (cf. Figure 2). One obvious reason for doing this is the relative simplicity of these paths, which allow for explicit computations of the (asymptotically) preferred angles of propagation as $l \rightarrow 0^{+}$. On the other hand, the small segments should approximate any rectifiable curve.

As we will see later, $-\Delta E(l ; \alpha)$ (the parameter $\alpha$ is used now to indicate the trial path) can be expanded in fractional powers of $l$ :

$$
-\Delta E(l ; \alpha)=G(\alpha) l+H(\alpha) l^{3 / 2}+O\left(l^{2}\right),
$$

for a suitable function $H(\alpha)$. Our main objective is to estimate the optimal angle $\alpha(l)$ for $l \rightarrow 0^{+}$, and in this way to establish a discrete crack propagation law in terms of the local parameters defining the elastic field. In the following we give a derivation of these results.

\section{General facts about Mode III fields.}

3.1. The boundary value problem. Consider a three dimensional body given by $\Omega \times \mathbb{R}:=\left\{\left(x_{1}, x_{2}, x_{3}\right):\left(x_{1}, x_{2}\right) \in \Omega, x_{3} \in \mathbb{R}\right\}$, where $\Omega$ is a domain in $\mathbb{R}^{2}$ with piecewise smooth boundary. This setting is typical for Mode III, where the invariance of the domain with respect to $x_{3}$ allows for some simplifications of the problem at hand. We recall the equilibrium equations of elasticity in the absence of body forces: 


$$
\sigma_{i j, j}=0, \quad 1 \leq i, j \leq 3,
$$

where $\sigma$ is the stress tensor and the summation convention is assumed. The tensor $\sigma$ is linked to the strain tensor $\varepsilon$ by the constitutive equations:

$$
\sigma_{i j}=\lambda \delta_{i j} \operatorname{tr}(\varepsilon)+2 \mu \varepsilon_{i j},
$$

where tr stands for the trace and $\lambda, \mu$ are the so called Lamé coefficients. For small deformations, $\varepsilon$ is given in terms of the displacement gradient as follows:

$$
\varepsilon_{i j}:=\frac{1}{2}\left(u_{i, j}+u_{j, i}\right) .
$$

The boundary conditions are given by:

$$
u_{i}\left(x_{1}, x_{2}, x_{3}\right)=D_{i}\left(x_{1}, x_{2}\right), \quad\left(x_{1}, x_{2}\right) \in \partial_{D} \Omega,
$$

in terms of the displacements, and by:

$$
\sigma_{i j} n_{j}\left(x_{1}, x_{2}, x_{3}\right)=T_{i}\left(x_{1}, x_{2}\right), \quad\left(x_{1}, x_{2}\right) \in \partial_{T} \Omega,
$$

for the normal stresses. Notice that $\partial_{D} \Omega \cup \partial_{T} \Omega=\partial \Omega$ and $n$ is a unit normal to $\partial \Omega$.The fields $D$ and $T$ represent the prescribed displacements and tractions at the boundary. For pure Mode III they further satisfy:

$$
D_{i} \equiv 0, T_{i} \equiv 0, \quad i=1,2 .
$$

Taking into account that the boundary conditions are independent of $x_{3}$ and due to the symmetry properties of the domain we can assume (by uniqueness) that the fields involved are independent of $x_{3}$ too. Then we can write:

$$
\begin{aligned}
\sigma_{i 3} & \equiv \sigma_{3 i}=\mu u_{3, i} \quad \text { for } i=1,2, \\
\sigma_{33} & =\lambda\left(u_{1,1}+u_{2,2}\right) .
\end{aligned}
$$

The equilibrium equation for $i=3$ in (3.1) is given by:

$$
\sigma_{31,1}+\sigma_{32,2}=0 .
$$

Using the constitutive relationship (3.2) we have that:

$$
\mu\left(u_{3,11}+u_{3,22}\right)=0,
$$

stating that $u_{3}$ is a harmonic function in $\Omega$. The condition for the out of plane component of the force is given by (notice that $n_{3} \equiv 0$ ):

$$
\sigma_{31} n_{1}+\sigma_{32} n_{2}=T_{3}, \text { on } \partial_{T} \Omega,
$$

and taking into account equation (3.3) we have:

$$
u_{3,1} n_{1}+u_{3,2} n_{2} \equiv \frac{\partial u_{3}}{\partial n}=\frac{1}{\mu} T_{3} \text { on } \partial_{T} \Omega .
$$

The field $u_{3}$ is then uncoupled from the other components.

Notation: From now on we will denote $u\left(x_{1}, x_{2}\right) \equiv u_{3}\left(x_{1}, x_{2}, x_{3}\right)$ for the out of plane displacement field. Moreover, we will drop the index 3 in the components of the stresses and boundary data, ie. we will write $T$ for $T_{3}, D$ for $D_{3}$ (we recall that the other components of the boundary data are zero) and $\sigma_{j}$ for $\sigma_{3 j}$. 
3.2. The moving boundary value problem. Let us assume that $(0,0) \in \Omega$. Consider a piecewise smooth Jordan curve $\Gamma_{0}$ in $\Omega$ as initial crack configuration such that one of its ends lies in $\partial \Omega$ and the other one is located at the origin. We impose now zero normal stress on the surface $\Gamma_{0} \times \mathbb{R}$ :

$$
\sigma_{1} n_{1}+\sigma_{2} n_{2}=0, \text { on } \Gamma_{0},
$$

which in turn means that (cf.(3.3)):

$$
\mu u_{, 1} n_{1}+\mu u_{, 2} n_{2} \equiv \mu \frac{\partial u}{\partial n}=0 \text { on } \Gamma_{0},
$$

We then include the Neumann homogeneous type boundary condition for a growing crack $\Gamma$ defining in this way a moving boundary problem for the out of plane field $u\left(x=\left(x_{1}, x_{2}\right)\right)$ :

$$
\begin{aligned}
u_{, 11}+u_{, 22} & =0, & & x \in \Omega \backslash \Gamma, \\
u(x) & =D(x), & & x \in \partial_{D} \Omega, \\
\frac{\partial u}{\partial n}(x) & =T(x), & & x \in \partial_{T} \Omega, \\
\frac{\partial u}{\partial n}(x) & =0 & & x \in \Gamma,
\end{aligned}
$$

3.3. Energy variation for a crack extension. The main ingredient of our approach is an expression for the energy variations for different crack configurations. It is then necessary to consider solutions $u \in H^{1}(\Omega \backslash \Gamma)$, or in other words, with integrable energy. Consider an extension of the curve $\Gamma_{0}$ given by a piecewise smooth curve $\Gamma_{l}$ indexed by arc extension length, such that:

$$
\Gamma_{l_{1}} \subset \Gamma_{l_{2}} \quad 0 \leq l_{1} \leq l_{2} .
$$

The mechanical energy functional for each $l \geq 0$ is given by:

$$
E(l):=\frac{\mu}{2} \int_{\Omega_{l}}\left|\nabla u_{l}\right|^{2}-\int_{\partial_{T} \Omega} T u_{l},
$$

where $\Omega_{l}:=\Omega \backslash \Gamma_{l}$. We define the incremental quantities:

$$
\begin{aligned}
\Delta E & :=E(l)-E(0), \\
v(x) & :=u_{l}(x)-u_{0}(x) \quad x \in \Omega_{l} \subset \Omega_{0} \\
\Delta \Gamma & :=\Gamma_{l} \backslash \Gamma_{0} .
\end{aligned}
$$

Notice that $v=0$ on $\partial_{D} \Omega$ and $\frac{\partial v}{\partial n}=0$ on $\partial_{T} \Omega$. We have that:

$$
\begin{aligned}
\Delta E & =\mu \int_{\Omega_{l}} \nabla u_{0} \cdot \nabla v+\frac{\mu}{2} \int_{\Omega_{l}} \nabla v \cdot \nabla v-\int_{\partial_{T} \Omega} T v \\
& =\frac{\mu}{2} \int_{\Delta \Gamma}\left(v^{+} \frac{\partial u_{0}}{\partial n^{+}}+v^{-} \frac{\partial u_{0}}{\partial n^{-}}\right),
\end{aligned}
$$

where we used the identities (3.7)-(3.10) and the fact that $\int_{\Delta \Gamma^{ \pm}} v \frac{\partial u_{l}}{\partial n}=0$. On each side of the slit $\Delta \Gamma$ we have that $\vec{n}^{+}=-\vec{n}^{-}$, and then we can write:

$$
\Delta E=\frac{\mu}{2} \int_{\Delta \Gamma}[v] \frac{\partial u_{0}}{\partial n^{+}},
$$

where the integration is taken with respect to the arc-length parameter and $[v]:=$ $v^{+}-v^{-}$is the jump of the field $v$ across the curve $\Delta \Gamma$. Recalling the fact that $u_{0}$ 
is continuous along $\Delta \Gamma$ we have $[v]=\left[u_{l}\right]$, and then we write the final expression for the mechanical energy increment in pure Mode III as follows:

$$
\Delta E=\frac{\mu}{2} \int_{\Delta \Gamma}\left[u_{l}\right] \frac{\partial u_{0}}{\partial n^{+}} .
$$

Notice that (3.12) is valid for finite extensions and for arbitrary shapes of $\Delta \Gamma$.

3.4. The asymptotic field. We study now the local field around the crack tip. This is accomplished by neglecting the effects of the curvature of the pre-existing crack and the particular geometry of the body, assuming that the tip is far from the boundary. We will see later that this is not an over-simplification of the problem: up to certain order in the field expansion the coefficients are universal (they do not depend explicitly on the particular geometry). This is shown by Amestoy and Leblond in [1] and we will go back to this point later. Let us take:

$$
\Omega \equiv \mathbb{R}^{2}, \quad \Gamma_{0} \equiv\left\{\left(x_{1}, 0\right): x_{1} \leq 0\right\} .
$$

Given an extended crack $\Gamma_{l} \supset \Gamma_{0}$ we consider a field $u_{l}$ satisfying the equilibrium equations:

$$
\begin{aligned}
u_{l, 11}+u_{l, 22} & =0, & x & \in \mathbb{R}^{2} \backslash \Gamma_{l}, \\
\frac{\partial u_{l}}{\partial n}(x) & =0, & & x \in \Gamma_{l} .
\end{aligned}
$$

Additionally, we require that $u_{l}$ belongs to the class:

$$
\mathcal{C}_{l}:=\cap_{r>0} H^{1}\left(B_{r}(0) \backslash \Gamma_{l}\right),
$$

where $B_{r}(0)$ is the disc of radius $r$, centered at the origin. This condition guarantees the existence of $\Delta E$ in (3.12). Nevertheless, the field $u_{l}$ is still not well defined, we must impose the matching condition with the initial equilibrium field:

$$
\lim _{x \rightarrow \infty}\left|u_{l}(x)-u_{0}(x)\right|=0,
$$

uniformly in $x$, where $u_{0}$ is a given scalar field satisfying the equilibrium equation (3.14), the boundary condition (3.15) for $l=0$, and belonging to the class $\mathcal{C}_{0}$ defined in (3.16).

Remark 1. It is worth to keep in mind that the condition (3.17) at infinity is more general than the typical boundary data used in the engineering literature. It is possible to find closed form solutions for a given uniform stress at infinity (cf. [17]) which are of limited applicability to the problem at hand. Notice that, with (3.17) we keep all the information given by the local field around the tip.

The uniqueness of the field $u_{l}$ can be established easily if we transform the set $\mathbb{R}^{2} \backslash \Gamma_{l}$ into the upper half plane by conformal mapping. Considering now two solutions $u_{l}^{1}$ and $u_{l}^{2}$, we have that the transformed difference $\Delta u=u_{l}^{1}-u_{l}^{2}$ is harmonic in the upper half plane and satisfies Neumann homogeneous conditions on the real line. By Schwarz reflection we obtain a harmonic function in the whole plane with uniform limit 0 at infinity. This function is bounded, since it belongs to the class $\mathcal{C}_{l}$. The uniqueness then follows from Liouville theorem. Later on, we will give explicit conformal maps for the relevant crack extensions. Notice that we have the freedom to select $u_{0}$ inside a wide class of functions. Once $u_{0}$ and $\Gamma_{l}$ are selected, the whole family $u_{l}$ is uniquely defined. 
3.5. Complex representation and expansion of the field around the tip. Notation: We denote by $\mathbb{C}$ the set of complex numbers and $\mathbb{C}^{*}:=\mathbb{C} \cup\{\infty\}$ the extended complex plane. $\mathbb{H}$ is the upper half plane including the real line and $\mathbb{H}^{+}, \mathbb{H}^{-}$are the $z \in \mathbb{C}$ such that $\operatorname{Im} z>0$ and $\operatorname{Im} z<0$ respectively.

Consider now equations (3.3) and (3.4) for the stress components:

$$
\begin{aligned}
& \sigma_{1,1}=-\sigma_{2,2}, \\
& \sigma_{1,2}=\sigma_{2,1} .
\end{aligned}
$$

These are the Cauchy-Riemann equations for the scalar functions $\sigma_{1}$ and $-\sigma_{2}$. There exists a complex function $\eta$, analytic in $\Omega \backslash \Gamma$, such that:

$$
\mu \eta^{\prime}(\zeta)=\sigma_{1}-i \sigma_{2}, \quad \zeta=x_{1}+i x_{2},
$$

and moreover:

$$
\operatorname{Re} \eta(\zeta)=u .
$$

For $\Gamma=\Gamma_{0}(\Omega \equiv \mathbb{C})$ we can apply the conformal map

$$
\zeta=f_{0}(z):=-z^{2}
$$

which sends $z \in \mathbb{H}^{+}$into $\mathbb{C} \backslash \Gamma_{0}$. We denote by $h_{0}$ the composition of $\eta_{0}$ and $f_{0}$, to emphasize the change of domain:

$$
h_{0}(z):=\eta_{0}\left(-z^{2}\right), \quad \operatorname{Im} z>0 .
$$

Now the boundary conditions take the form:

$$
\operatorname{Im} h_{0}^{\prime}(z)=0, \quad \text { for } \operatorname{Im}(z)=0 .
$$

Applying Schwarz's symmetry principle (cf. [15]) we can extend analytically $h_{0}^{\prime}$ to the lower half plane by means of:

$$
h_{0}^{\prime}(z)=\overline{h_{0}^{\prime}(\bar{z})} .
$$

Notice that (3.16) rules out any singularity around the origin. Therefore, $h_{0}$ admits an expansion with real coefficients:

$$
h_{0}(z)=\sum_{n=0} c_{n} z^{n}, \quad c_{n} \in \mathbb{R}, z \in \mathbb{C}
$$

and the following expansion is valid in the original domain:

$$
\eta_{0}(\zeta)=\sum_{n=0} c_{n}(-\zeta)^{n / 2}, \quad c_{n} \in \mathbb{R}, \zeta \in \mathbb{C} \backslash \Gamma_{0} .
$$

Taking real part and then polar coordinates this expansion turns to be:

$$
u_{0}=\operatorname{Re}\left(\sum_{n=0} c_{n}(\sqrt{-\zeta})^{n}\right)=c_{0}-c_{1} r^{1 / 2} \sin (\theta / 2)-c_{2} r \cos (\theta)+\ldots
$$

The coefficient for $r^{1 / 2} \sin (\theta / 2)$ is (up to a multiplicative constant) the so-called stress intensity factor for Mode III loading:

$$
c_{1}=-\frac{1}{\mu} \sqrt{\frac{2}{\pi}} K_{\mathrm{III}} .
$$

This term contains the leading behavior of the field as $r \rightarrow 0$. The asymptotic relationship (3.27) is the typical expression for the Mode III field around the tip of a smooth crack (cf. Freund [10]). It is usually obtained by dimensional arguments and asymptotic analysis. 
The basic crack configurations that we will use are given by kinked paths (cf. Figure $2)$. Given an angle $\varphi$, with $-\pi \leq \varphi \leq \pi$, and $l \geq 0$ we take:

$$
\Gamma_{l}:=\Gamma_{0} \cup\left\{\left(x_{1}, x_{2}\right): x_{1}=r \cos \varphi, x_{2}=r \sin \varphi, 0 \leq r \leq l\right\} .
$$

As above, we denote by $\Delta \Gamma$ the segment defining the extension $\Gamma_{l} \backslash \Gamma_{0}=\left\{\left(x_{1}, x_{2}\right)\right.$ : $\left.x_{1}=r \cos \varphi, x_{2}=r \sin \varphi, 0 \leq r \leq l\right\}$. If we re-scale the domain with respect to the parameter $l$, the shape of the kink will remain in the limit. Therefore, the expansion (3.27) is not accurate near the corner of the path. In order to compute the energy increments (cf. (3.12)) we need a precise value of the field along the whole segment $\Delta \Gamma$. By using the same argument as above, we introduce a new expansion using a family of conformal maps, indexed by the parameters $\alpha$ and $l$ (see the Appendix):

$$
f_{l}(z):=-(z-a(l))^{1-\alpha}(z-b(l))^{1+\alpha},
$$

where $\alpha:=\varphi / \pi(-1 \leq \alpha \leq 1)$ and:

$$
a(l):=-\sqrt{l}\left(\frac{1-\alpha}{1+\alpha}\right)^{\frac{1+\alpha}{2}}, \quad b(l):=\sqrt{l}\left(\frac{1+\alpha}{1-\alpha}\right)^{\frac{1-\alpha}{2}},
$$

The function given in (3.29) sends the upper half plane $\mathbb{H}^{+}$onto the set $\mathbb{R}^{2} \backslash \Gamma_{l}$, where the curves $\Gamma_{l}$ are defined in (3.28). Moreover $f_{l}(\mathbb{R})=\Gamma_{l}$ and $f_{l}([a(l), b(l)])=\Delta \Gamma$. See Figure 2 below. Under the same arguments that lead to the expansion (3.25),

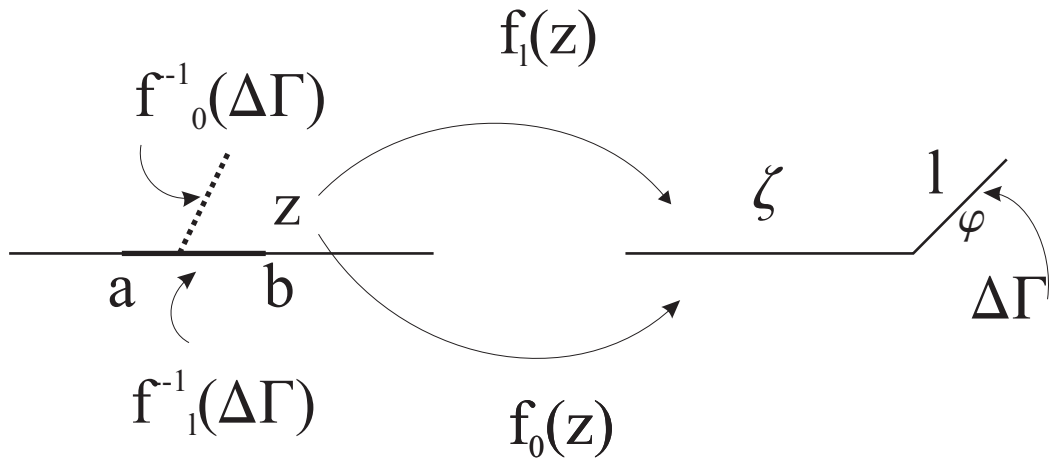

FiguRE 2. The mapping properties of $f_{l}$ and $f_{0}$.

we obtain for $\Gamma=\Gamma_{l}$ that the solution $u_{l}$ is the real part of some complex function $\eta_{l}$. Moreover, writing:

$$
\zeta=f_{l}(z)
$$

we define:

$$
h_{l}(z):=\eta_{l}\left(f_{l}(z)\right), \quad \operatorname{Im}(z)>0 .
$$

Elementary properties of conformal mapping combined with the Neumann homogeneous conditions satisfied by $\operatorname{Re} \eta_{l}(\zeta)$, give:

$$
\operatorname{Im} h_{l}^{\prime}(z)=0, \quad \text { for } \operatorname{Im}(z)=0 .
$$

By the symmetry principle the extended function

$$
h_{l}^{\prime}(z)=\overline{h_{l}^{\prime}(\bar{z})}, \quad z \in \operatorname{Im} z<0,
$$


is analytic in the whole plane. Therefore, $h_{l}$ admits an expansion of the form:

$$
h_{l}(z)=\sum_{n=0} c_{n}(l) z^{n}, \quad c_{n}(l) \in \mathbb{R} .
$$

In the original domain we must have the following identity:

$$
\eta_{l}(\zeta)=\sum_{n=0} c_{n}(l)\left(f_{l}^{-1}(\zeta)\right)^{n}, \quad c_{n} \in \mathbb{R}, \zeta \in \mathbb{C} \backslash \Gamma_{l}
$$

Notice that for $l=0$ and for any $\varphi$, we have that $a(0)=b(0)=0$, then we have that:

$$
f_{0}(z)=-z^{2}
$$

and the expansion (3.26) for $\eta_{0}$ is recovered. We prove now the following simple result.

Proposition 1. The field $u_{l}$ satisfying (3.14)-(3.17) is given by the real part of the non negative powers in the Laurent representation of $g_{l}(z):=\eta_{0}\left(f_{l}(z)\right)$ around the origin, namely:

$$
u_{l}\left(f_{l}(z)\right)=\operatorname{Re} \frac{1}{2 \pi i} \int_{C} \frac{g_{l}(\zeta)}{\zeta-z} d \zeta,
$$

where $C$ is a curve enclosing $z$ and the interval $I_{l}:=[a(l), b(l)]$ is defined in (3.30).

Proof. The real function given by:

$$
v_{l}(x):=u_{l}(x)-u_{0}(x)
$$

is harmonic in $\mathbb{R}^{2} \backslash \Gamma_{l}$. By the matching condition (3.17) it goes to zero uniformly at infinity. By (3.15) satisfies homogeneous Neumann boundary conditions on $\Gamma_{0}$ and

$$
\frac{\partial v_{l}}{\partial n}(x)=-\frac{\partial u_{0}}{\partial n}(x) \quad \text { for } x \in \Delta \Gamma .
$$

If we look at this function through the map $f_{l}(z)$, we have that $v_{l}\left(f_{l}(z)\right)$ is harmonic in the upper half plane, it goes to zero at infinity, satisfies homogeneous Neumann boundary conditions on $\mathbb{R} \backslash I_{l}$ and non homogeneous Neumann conditions on $I_{l}$. It can therefore be extended by symmetry to a harmonic function in $\mathbb{C} \backslash I_{l}$. By analytic completion of the Poisson formula for the upper half plane we have that:

$$
v_{l}\left(f_{l}(z)\right)=\operatorname{Re} p_{l}(z)
$$

where

$$
\begin{aligned}
& p_{l}(z):=\frac{1}{\pi} \int_{a(l)}^{b(l)} \frac{r_{l}(t)}{t-z} d t, \\
& r_{l}(x)=-\int_{a(l)}^{x} \operatorname{sig}(t) \frac{\partial u_{0}}{\partial n}\left(f_{l}(t)\right)\left|f_{l}^{\prime}(t)\right| d t \quad a(l) \leq x \leq b(l),
\end{aligned}
$$

where $\operatorname{sig}(t)=-1$ for $t<0$ and $\operatorname{sig}(t)=+1$ for $t>0$. Notice that $r_{l}(b(l))=0$. The function $p_{l}$ is holomorphic in $\mathbb{C}^{*} \backslash I_{l}$ (recall that $\mathbb{C}^{*}:=\mathbb{C} \cup\{\infty\}$ ) and $p_{l}(\infty)=0$. It therefore admits a Laurent expansion for $|z|>R>\max \{-a(l), b(l)\}$ with strictly negative powers of $z$. Using the notation introduced in (3.31), we have that:

$$
h_{l}(z)=g_{l}(z)+p_{l}(z),
$$

where $g_{l}(z):=\eta_{0}\left(f_{l}(z)\right)$. As $h_{l}$ is analytic in $\mathbb{C}(\mathrm{cf} .3 .32)$ we must have that $g_{l}$ and $p_{l}$ share the negative powers in $z$ (with opposite sign) in their respective Laurent 
expansions around $z=0$. Therefore, if the curve $C$ encloses $z$ and $I_{l}$, we must have that:

$$
h_{l}(z)=\frac{1}{2 \pi i} \int_{C} \frac{g_{l}(\zeta)}{\zeta-z} d \zeta
$$

and (3.34) follows.

Corollary 1. The coefficients $c_{n}(l)$ in (3.32) satisfy the following relationship:

$$
c_{n}(l)=c_{n}+(n+1) c_{n+1} b_{0}(l)+O(l),
$$

where $b_{0}(l)=\sqrt{l} b_{0}(1)$ is the constant term in the Laurent expansion for $\chi_{l}:=$ $f_{0}^{-1} \circ f_{l}$ as $z \rightarrow \infty$.

Proof. The function $g_{l}(z)$ may be written as follows (cf. (3.22) and (3.23) above):

$$
g_{l}(z)=\eta_{0}\left(f_{l}(z)\right)=h_{0}\left(f_{0}^{-1} \circ f_{l}(z)\right) .
$$

We denote by $\chi_{l}$ the function $f_{0}^{-1} \circ f_{l}$, which sends the upper half plane in a one to one way to the upper half plane minus the set $f_{0}^{-1}(\Delta \Gamma)$. Notice that $\chi_{l}$ is real on $\mathbb{R} \backslash I_{l}$ and therefore can be extended by symmetry to an analytic univalent function on $\mathbb{C} \backslash I_{l}$. Moreover it admits an expansion of the form (cf. (3.29)):

$$
\begin{aligned}
\chi_{l}(z) & =\sqrt{(z-a(l))^{1-\alpha}(z-b(l))^{1+\alpha}} \\
& =z+b_{0}(l)+\frac{b_{1}(l)}{z}+\frac{b_{2}(l)}{z^{2}} \ldots \quad z \rightarrow \infty,
\end{aligned}
$$

Due to the following scaling property of $\chi_{l}$ :

$$
\chi_{l}(z)=\sqrt{l} \chi_{1}(z / \sqrt{l}),
$$

we have that:

$$
b_{n}(l)=l^{\frac{n+1}{2}} b_{n}(1) .
$$

We use now the formula for the coefficients in a Laurent expansion (cf. [15]), taking into account the series for $h_{0}$ given in (3.25) and (3.35):

$$
c_{n}(l)=\frac{1}{2 \pi i} \int_{C} \frac{g_{l}(\zeta)}{\zeta^{n+1}} d \zeta=\frac{1}{2 \pi i} \int_{C} \frac{h_{0}\left(\chi_{l}(\zeta)\right)}{\zeta^{n+1}} d \zeta=\frac{1}{2 \pi i} \sum_{j=0}^{\infty} c_{j} \int_{C} \frac{\chi_{l}^{j}(\zeta)}{\zeta^{n+1}} d \zeta
$$

From (3.37) we obtain:

$$
c_{n}(l)=\frac{1}{2 \pi i} \sum_{j=0}^{\infty} c_{j} \int_{C} \frac{d \zeta}{\zeta^{n+1}}\left(\zeta+\sum_{k=0}^{\infty} \frac{b_{k}(l)}{\zeta^{k}}\right)^{j} .
$$

We see at once that:

$$
\int_{C} \frac{d \zeta}{\zeta^{n+1}}\left(\zeta+\sum_{k=0}^{\infty} \frac{b_{k}(l)}{\zeta^{k}}\right)^{j}=0 \text { for } j<n
$$

and then we can write:

$$
c_{n}(l)=\frac{1}{2 \pi i} \sum_{j=n}^{\infty} c_{j} \int_{C} \frac{d \zeta}{\zeta^{n+1}}\left(\zeta+\sum_{k=0}^{\infty} \frac{b_{k}(l)}{\zeta^{k}}\right)^{j} .
$$

For $j=n$ the contribution inside the parentheses is given by $\zeta^{n}$, giving the value $c_{n}$. For $j=n+1$ we have the contribution of $\zeta^{n} b_{0}(l)$ giving rise to $(n+1) c_{n+1} b_{0}(l)$ 
(cf. (3.39)). The next one gives, for $j=n+2$, the term $c_{n+2}\left(\left(\begin{array}{c}n+2 \\ 2\end{array}\right) b_{0}^{2}(l)+(n+2) b_{1}(l)\right)$, which is of order $l$. It is easy to see that further terms give corrections of order $l^{3 / 2}$ and higher.

Remark 2. Formula (3.36) shows an expansion for all the coefficients of the field $u_{l}$ in terms of $l$, not only for the "stress intensity factor" $c_{1}$. Nevertheless, in the case of finite domains or curved initial configurations, the term of order $l$ should include a non universal function, as it is shown by Leblond in [14].

3.6. A complex-variable formula for the energy variation. It is useful to express the energy increment in (3.12) by means of a complex integral in the transformed domain $z$. Let us recall the complex representation of the stresses (3.20) and displacements (3.21):

$$
\sigma_{1}^{l}-i \sigma_{2}^{l}=\mu \eta_{l}^{\prime}(\zeta), \quad u_{l}(\zeta)=\operatorname{Re} \eta_{l}(\zeta) .
$$

Notice the superscript $l$ indicating the state of stress corresponding to displacement $u_{l}$. If we rotate the axes an angle $\theta$, the tangential and normal components of the stress change as follows:

$$
\sigma_{t}^{l}-i \sigma_{n}^{l}=\mu \eta_{l}^{\prime}(\zeta) e^{i \theta}
$$

This can be easily seen by applying the force balance to a triangle with an appropriate orientation. It is worth to mention that $\sigma_{t}^{l}$ represents the out of plane stress component (ie., in the $x_{3}$ direction) when we take a face with normal in direction $\theta$. Similarly, $\sigma_{n}^{l}$ is the out of plane stress component when we consider a face with normal in the direction of $\theta+\pi / 2$. Our purpose is to give an expression for the line integral:

$$
\Delta E=\frac{1}{2} \int_{\Delta \Gamma}\left[u_{l}\right]\left(\mu \frac{\partial u_{0}}{\partial n^{+}}\right)=\frac{\mu}{2} \int_{\Delta \Gamma}\left[\operatorname{Re} \eta_{l}\right] \operatorname{Im}\left(\eta_{0}^{\prime} e^{i \varphi}\right) .
$$

Where $n^{+}$is the exterior normal to $\Delta \Gamma^{+}$, ie. with $\operatorname{argument} \varphi-\pi / 2$.

We apply now the conformal map $f_{l}$ as in (3.31). Recalling the definition of $g_{l}$ :

$$
g_{l}(z):=\left(\eta_{0} \circ f_{l}\right)(z),
$$

then:

$$
\mu g_{l}^{\prime}(z)=\mu \eta_{0}^{\prime}\left(f_{l}(z)\right) f_{l}^{\prime}(z)=\left(\sigma_{t}^{0}-i \sigma_{n}^{0}\right)\left(f_{l}(z)\right)\left|f_{l}^{\prime}(z)\right|,
$$

where now $t$ and $n$ are the directions given by the conformal transformation of directions $z_{1}$ and $z_{2}$ respectively. When $z$ approaches the real axis outside the interval $I_{l}:=[a(l), b(l)]$ (ie. $\left.z_{2} \rightarrow 0^{+}, z_{1} \in \mathbb{R} \backslash I_{l}\right), \sigma_{t}^{0}, \sigma_{n}^{0}$ represent the tangential and normal stress components along the initial crack $\Gamma_{0}$. Therefore, taking into account that $\sigma_{n}^{0} \rightarrow 0$ on $\Gamma_{0}$, we have that:

$$
\mu g_{l}^{\prime}(z)=\sigma_{t}^{0}\left(f_{l}(z)\right)\left|f_{l}^{\prime}(z)\right| \in \mathbb{R} \quad \text { for } z \in \mathbb{R} \backslash I_{l} .
$$

Let us now change the integration variable in (3.40) by means of $\zeta=f_{l}(z)$ :

$$
\begin{aligned}
\Delta E & =\frac{\mu}{2} \int_{a(l)}^{0} \operatorname{Re} h_{l} \operatorname{Im}\left(\eta_{0}^{\prime} e^{i \varphi}\right)\left|f_{l}^{\prime}\right| d z-\frac{\mu}{2} \int_{0}^{b(l)} \operatorname{Re} h_{l} \operatorname{Im}\left(\eta_{0}^{\prime} e^{i \varphi}\right)\left|f_{l}^{\prime}\right| d z \\
& =\frac{\mu}{2} \int_{a(l)}^{b(l)} \operatorname{Re} h_{l} \operatorname{Im} g_{l}^{\prime} d z .
\end{aligned}
$$


where we recall that $h_{l}=\eta_{l} \circ f_{l}$ (cf.(3.31) and (3.41)), $f_{l}([a(l), 0])=\Delta \Gamma^{+}$and $f_{l}([0, b(l)])=\Delta \Gamma^{-}$(cf.(3.30)). Notice also that $f_{l}^{\prime}(z)=e^{i \varphi}\left|f_{l}^{\prime}(z)\right|$ for $a(l)<z<$ 0 , and $f_{l}^{\prime}(z)=e^{i(\varphi+\pi)}\left|f_{l}^{\prime}(z)\right|$ for $0<z<b(l)$.

Using the boundary conditions we have that:

$$
h_{l}(z) \in \mathbb{R} \quad \text { for } z \in \mathbb{R} .
$$

On the other hand we can extend the function $g_{l}$ to the lower half plane:

$$
g_{l}(z)=\overline{g_{l}(\bar{z})}, \quad z \in \operatorname{Im} z<0 .
$$

By (3.42) this function is real on $\mathbb{R} \backslash I_{l}$ and we have that the extended $g_{l}$ is sectionally holomorphic, ie. it is analytic in the plane except on the segment $I_{l}$ (cf.(3.30)).

Using now (3.43), (3.44) and the fact that the extended $h_{l}(z)$ is analytic in $\mathbb{C}$ and real for $z \in \mathbb{R}$, we obtain:

$$
\Delta E=\frac{\mu}{2} \int_{a(l)}^{b(l)} h_{l} \operatorname{Im} g_{l}^{\prime} d z=\frac{\mu i}{4} \int_{C} h_{l} g_{l}^{\prime} d z,
$$

where $C$ is a closed curve in the complex plane surrounding the real interval $I_{l}$. After integrating by parts we find that:

$$
\Delta E=\frac{\mu}{4 i} \int_{C} h_{l}^{\prime} g_{l} d z
$$

Notice that for $l=0, g_{0}$ reduces to $h_{0}$, the integrand being an analytic function and the result is therefore zero, as expected.

\section{THE CRACK PATH.}

4.1. An expansion for $\Delta E$. To clarify the dependence on $l$, we write (3.45) as follows (cf.(3.41)):

$$
\Delta E=\frac{\mu}{4 i} \int_{C} h_{l}^{\prime}(z) h_{0}\left(\chi_{l}(z)\right) d z .
$$

This shows more explicitly the role of the conformal maps $\chi_{l}=f_{0}^{-1} \circ f_{l}: \mathbb{H} \mapsto \mathbb{H}$. Let us insert the expansions (3.25) and (3.32) in (4.1):

$$
\Delta E=\frac{\mu}{4 i} \int_{C}\left(\sum_{j=1} j c_{j}(l) z^{j-1}\right)\left(\sum_{k=0} c_{k}\left(\chi_{l}(z)\right)^{k}\right) d z .
$$

For $k=0$ in the second sum, the integrand is holomorphic and there is no contribution to the integral. Using the scale invariance property $\chi_{l}(z)=\sqrt{l} \chi_{1}(z / \sqrt{l})$ (cf. (3.38)) we find:

$$
\Delta E=\frac{\mu}{4 i} \int_{C}\left(\sum_{j=1} j c_{j}(l) z^{j-1}\right)\left(\sum_{k=1} l^{k / 2} c_{k}\left(\chi_{1}(z / \sqrt{l})\right)^{k}\right) d z .
$$

After the change of variables $z=\sqrt{l} w$, this turns to be:

$$
\Delta E=\frac{\mu}{4 i} \int_{C}\left(\sum_{j=1} l^{\frac{j}{2}} j c_{j}(l) w^{j-1}\right)\left(\sum_{k=1} l^{\frac{k}{2}} c_{k}\left(\chi_{1}(w)\right)^{k}\right) d w .
$$

Notice that we can keep the same integration path if $C$ encloses the segment $[a(1), b(1)]$ with positive orientation. 
4.2. The energy release rate. We look now for the first term in the expansion in powers of $l$. Taking $j, k=1$ we see that the lower exponent for $l$ has the value 1 , and together with (3.36), (3.37) and (3.39) gives:

$$
\begin{aligned}
\Delta E & =\frac{l \mu}{4 i} c_{1} c_{1}(l) \int_{C} \chi_{1}(w) d w+O\left(l^{3 / 2}\right) \\
& =\frac{l \mu}{4 i} c_{1}^{2} \int_{C}\left(b_{0}(1)+\frac{b_{1}(1)}{w}+\ldots\right) d w+O\left(l^{3 / 2}\right),
\end{aligned}
$$

where $C$ is a suitable closed path. The coefficient $b_{1}(1)$ is computed explicitly from (3.29) and (3.30), giving the value:

$$
b_{1}(1)=-\frac{1}{2}\left(\frac{1-\alpha}{1+\alpha}\right)^{\alpha}
$$

where we recall that $\alpha=\varphi / \pi, \varphi$ being the kinking angle. After integrating, we have that:

$$
\Delta E=-\frac{l \mu \pi}{4} c_{1}^{2}\left(\frac{1-\alpha}{1+\alpha}\right)^{\alpha}+O\left(l^{3 / 2}\right) .
$$

This expression shows us immediately the preferred direction of motion. For small $l$, the amount of elastic energy per unit length that the body can release is determined by the linear term in the expansion for $\Delta E$, whose negative value is the Energy Release Rate defined in (1.1):

$$
G(\alpha)=-\lim _{l \rightarrow 0^{+}} \frac{\Delta E}{l}=\frac{\mu \pi}{4} c_{1}^{2}\left(\frac{1-\alpha}{1+\alpha}\right)^{\alpha} .
$$

Notice that $G(\alpha)>0$ for all $\alpha$ such that $-1<\alpha<1$. Starting with $c_{1}=0$, and increasing slowly the loading to raise the stress around the tip (and therefore enlarging $\left.\left|c_{1}\right|\right)$ there will be a minimum critical value of $\left|c_{1}\right|$ which will make $G(\alpha)=$ $\kappa$ for some angle $\alpha$ (we recall that $\kappa$ is the surface energy per unit length, a material constant). This threshold will be reached when the universal function:

$$
A(\alpha):=\left(\frac{1-\alpha}{1+\alpha}\right)^{\alpha}
$$

attains its maximum value, which is achieved for $\alpha=0$ independently of the applied loading. The critical value of $\left|c_{1}\right|$ turns to be:

$$
\left|c_{1}\right|_{\text {crit }}=\sqrt{\frac{4 \kappa}{\mu \pi}}
$$

If $\left|c_{1}\right|<\left|c_{1}\right|_{\text {crit }}$ the motion of the tip is not possible, since there is not enough elastic energy available to pay for the surface energy. If $\left|c_{1}\right|>\left|c_{1}\right|_{\text {crit }}$ there is an excess of energy stored in the body and if the crack starts to grow it will generate dynamic effects (sound waves). If we remain in a quasistatic growth regime, we must have $\left|c_{1}\right| \approx\left|c_{1}\right|_{\text {crit }}$ and there is only one direction satisfying the balance of energy, namely $\alpha=0$. This shows that the motion of a Mode III crack will always follow the tangent to the initial configuration in a first approximation. In other words, for a pure out of plane loading, it would not be possible to generate a kink in the path in a quasistatic regime. As we already mentioned, this is in contrast with the in-plane modes where a kink is predicted for $K_{\text {II }} \neq 0$ (cf. for instance [6] and [14]). A plot of $A(\alpha)$ is shown in Figure (3). 


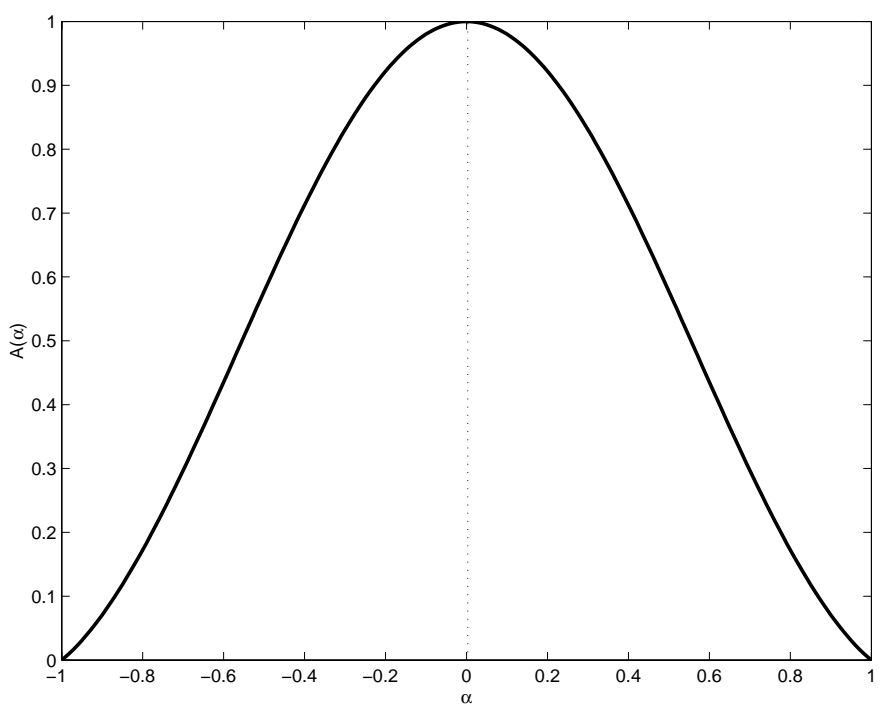

Figure 3. The function $A(\alpha)$.

It turns out that the linear term in the expansion of $\Delta E$ does not give enough information about the initial shape of the growing crack. Moreover, any smooth configuration of $\Gamma$ would satisfy the principle of "maximum energy release rate" at each point. We must therefore look for the next term, which is proportional to $l^{3 / 2}$. We note on pass that (4.6) can also be obtained by means of Irwin type formulae (cf. [13]) once the stress intensity factor for the kinked configuration is available. This factor was already computed by some authors, using integral equation methods and conformal mapping techniques (cf. for instance, formula 4.16 in [19]).

4.3. The second term in the expansion. Consider (4.3) once more, and notice that the next power for $l$ is $3 / 2$. The contribution to this term is given by all the pairs $j, k$ satisfying $j+k=3$ and also by $j, k=1$ from the first correction term of the coefficient $c_{1}(l)=c_{1}+2 \sqrt{l} c_{2} b_{0}(1)+O(l)$ (cf. (3.36) and (3.39)).

Case $1 j=1, k=1$ in (4.3) and (3.36).

$$
\frac{\mu}{4 i} \int_{C} l c_{1}(l) c_{1} \chi_{1}(w) d w=-l G(\alpha)+l^{3 / 2} \mu \pi c_{1} c_{2} b_{0}(1) b_{1}(1)+O\left(l^{2}\right) .
$$

We can compute explicitly $b_{0}(1)$ from (3.29) and (3.30) obtaining:

$$
b_{0}(1)=-\left(\frac{1-\alpha}{1+\alpha}\right)^{\frac{\alpha}{2}} \frac{2 \alpha}{\sqrt{1-\alpha^{2}}} .
$$

Together with (4.4) this gives (cf. (4.7)):

$$
\frac{\mu}{4 i} \int_{C} l c_{1}(l) c_{1} \chi_{1}(w) d w=-l G(\alpha)+l^{3 / 2} \mu \pi c_{1} c_{2} \frac{\alpha}{\sqrt{1-\alpha^{2}}} A(\alpha)^{\frac{3 \alpha}{2}}+O\left(l^{2}\right) .
$$


Case $2 j=1, k=2$ in (4.3). The contribution to the integral is given by the expression:

$$
\frac{\mu}{4 i} \int_{C} l^{\frac{3}{2}} c_{1}(l) c_{2}\left(\chi_{1}(w)\right)^{2} d w=\frac{\mu}{2 i} l^{\frac{3}{2}} c_{1} c_{2} \int_{C} \frac{b_{2}(1)+b_{0}(1) b_{1}(1)}{w} d w+O\left(l^{2}\right)
$$

where we used again (3.37). The coefficient $b_{2}(1)$ is computed explicitly from (3.29), (3.30), giving the value:

$$
b_{2}(1)=-\frac{1}{3} \frac{\alpha}{\sqrt{1-\alpha^{2}}}\left(\frac{1-\alpha}{1+\alpha}\right)^{\frac{3 \alpha}{2}} .
$$

From (4.4), (4.9) and (4.11) we have:

$$
b_{2}(1)+b_{0}(1) b_{1}(1)=\frac{2}{3} \frac{\alpha}{\sqrt{1-\alpha^{2}}}\left(\frac{1-\alpha}{1+\alpha}\right)^{\frac{3 \alpha}{2}} .
$$

We then obtain the contribution (cf. (4.7)):

$$
\frac{\mu}{4 i} \int_{C} l^{\frac{3}{2}} c_{1}(l) c_{2}\left(\chi_{1}(w)\right)^{2} d w=\frac{2 \mu \pi}{3} l^{\frac{3}{2}} c_{1} c_{2} \frac{\alpha}{\sqrt{1-\alpha^{2}}} A(\alpha)^{\frac{3}{2}}+O\left(l^{2}\right)
$$

Case $3 j=2, k=1$ in (4.3).

$$
\begin{aligned}
\frac{\mu}{4 i} \int_{C} l^{3 / 2} 2 c_{1} c_{2}(l) w \chi_{1}(w) d w & =\frac{\mu}{2 i} l^{3 / 2} c_{1} c_{2} \int_{C} w \chi_{1}(w) d w+O\left(l^{2}\right) \\
& =\mu \pi l^{3 / 2} c_{1} c_{2} b_{2}(1)+O\left(l^{2}\right) .
\end{aligned}
$$

Using (4.11) we obtain:

$$
\frac{\mu}{4 i} \int_{C} l^{3 / 2} 2 c_{1} c_{2}(l) w \chi_{1}(w) d w=-\frac{\mu \pi}{3} l^{3 / 2} c_{1} c_{2} \frac{\alpha}{\sqrt{1-\alpha^{2}}} A(\alpha)^{\frac{3 \alpha}{2}}+O\left(l^{2}\right)
$$

We now gather the contributions given by $(4.10),(4.12),(4.13)$ which together with (4.5) yield the expression:

$$
\Delta E=-\frac{\mu \pi}{4} c_{1}^{2} A(\alpha) l+\frac{4 \mu \pi}{3} c_{1} c_{2} B(\alpha) l^{\frac{3}{2}}+O\left(l^{2}\right),
$$

where we defined the universal function $B$ as follows:

$$
B(\alpha):=\frac{\alpha}{\left(1-\alpha^{2}\right)^{1 / 2}} A(\alpha)^{\frac{3}{2}} .
$$

A plot of $B(\alpha)$ for $-1<\alpha<1$ is shown in Figure 4 .

Notice that $B(0)=0$. This is compatible with the fact that $\Delta E$ has a second derivative with respect to $l$ for $\alpha=0$ fixed (ie., for a straight motion). This is also a consequence (through Irwin's relationship) of the differentiability of the stress intensity factor for rectilinear paths. On the other hand, we can see that the energy released is bigger when the sign of the term of power $l^{3 / 2}$ is negative. For a positive $c_{1} c_{2}$ the tip would release more energy if it goes to the left. If the relative signs of $c_{1}$ and $c_{2}$ are such that $c_{1} c_{2}<0$, then it is better to choose a positive angle. We will make this statements more precise in the following Section. 


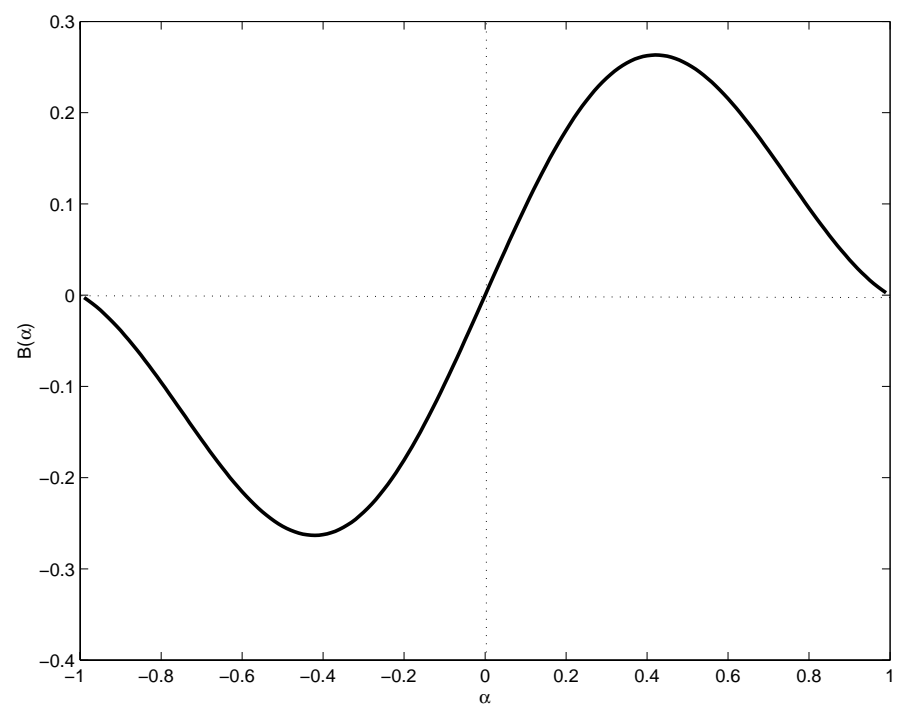

Figure 4. The universal function $B(\alpha)$ in the term of order $l^{3 / 2}$.

4.4. The approximate shape of the growing crack. Consider now the approximate expansion for the Energy increment:

$$
-\frac{\Delta E}{\mu \pi} \approx \frac{c_{1}^{2}}{4} A(\alpha) l-\frac{4 c_{1} c_{2}}{3} B(\alpha) l^{3 / 2} \quad l \rightarrow 0^{+} .
$$

An important property of this expression is that it is a universal expansion, in the sense that it is valid for a bounded body and for kinks starting from an arbitrary curved crack. To see this point it must be recalled that the expansion of the coefficient $c_{1}$ is used up to the term of order $\sqrt{l}$ (cf. (3.36)). As it is shown by Leblond, the first term containing information about the initial curvature and boundary geometry is the one of order $l$ (see sections 6 and 7 of [14]).

Let us now compute, for a small (but finite) $l$ the value $\alpha(l)$ that maximizes the right hand side. We know that this angle should be near zero because the first term has its maximum in that direction. With the aid of the term of order $l^{3 / 2}$ we will find the asymptotic correction to the rectilinear motion. To this end, consider the expansions of the analytic functions $A$ and $B$ around $\alpha=0$ :

$$
\begin{aligned}
& A(\alpha)=1-2 \alpha^{2}+O\left(\alpha^{4}\right), \\
& B(\alpha)=\alpha-\frac{5}{2} \alpha^{3}+O\left(\alpha^{4}\right)
\end{aligned}
$$

The derivatives are then given by:

$$
\begin{aligned}
& A^{\prime}(\alpha)=-4 \alpha+O\left(\alpha^{3}\right) \\
& B^{\prime}(\alpha)=1+O\left(\alpha^{2}\right) .
\end{aligned}
$$


We now gather the lower powers of $\alpha$ in (4.16) that cancel the following expression to the lowest order:

$$
\frac{c_{1}^{2}}{4}\left(-4 \alpha+O\left(\alpha^{3}\right)\right) l-\frac{4 c_{1} c_{2}}{3}\left(1+O\left(\alpha^{2}\right)\right) l^{3 / 2} .
$$

We see that $l \alpha$ should be balanced with $l^{3 / 2}$ giving as a result:

$$
c_{1}^{2} \alpha+\frac{4 c_{1} c_{2}}{3} l^{1 / 2}=o\left(l^{1 / 2}\right),
$$

or in other way:

$$
\lim _{l \rightarrow 0^{+}} \frac{\alpha}{l^{1 / 2}}=-\frac{4}{3} \frac{c_{2}}{c_{1}} .
$$

As we argued before, this shows that the sign of the initial angle is determined by the quotient $\frac{c_{2}}{c_{1}}$. In terms of the original coordinates $\left(x_{1}, x_{2}\right)$, we have that:

$$
x_{1}=l \cos (\pi \alpha) \approx l, \quad \frac{x_{2}}{x_{1}}=\tan (\pi \alpha) \approx \pi \alpha .
$$

We see that (4.17) is compatible with the following shape for the incipient crack extension:

$$
x_{2} \approx-\frac{4 \pi}{3} \frac{c_{2}}{c_{1}}\left(x_{1}\right)^{3 / 2}, \quad x_{1}>0 .
$$

Remark 3. Shapes as (4.18) were proposed a priori by Leblond to obtain behaviors compatible with the symmetry principle (cf.[14]). In our approach, this shapes appear as a consequence of the basic propagation law assumed.

4.5. A discrete algorithm of propagation. Following the results obtained in the previous Sections, we describe briefly an algorithm for the pure Mode III propagation of a quasistatic crack. This approach takes full account of the near tip field and selects the shape according to the discrete version of the maximum energy release rate criterium. On the other hand it does not violate condition (1.1) during the propagation process, unless the crack is already at rest.

Consider a domain $\Omega \subset \mathbb{R}^{2}$, an initial configuration of the crack $\Gamma_{0}$, smooth near its end tip, and boundary data indexed by a parameter $t \geq 0:(T(t), D(t))$, where $T$ indicates the normal stress, and $D$ is the given displacement as in (3.8)-(3.9). Given a natural number $N$, we proceed to divide the time interval into a finite sequence of time steps:

$$
0 \leq t_{j} \leq t^{\max }, \quad 0 \leq j \leq N .
$$

We should keep in mind that $t$ is not a "physical time" parameter, and there is nothing in the model connecting the time and length scales. Ideally, the time steps should be selected in such a way that the "energy excess" in a single step $j$, should be of the order of $t_{j+1}-t_{j}$. We will go back to this point later.

Given a value of $l>0$, the propagation is modelled as a discrete process, each step consisting of a kink of length $l$. The angle of kinking is selected according to the previous results. In each time-step, we can have several kink-steps, depending on the possibility of reaching a stable equilibrium point. We proceed to describe the algorithm. 
Definition 1. We say that a crack configuration $\Gamma_{j}$, at "time" step $j$, is in stable equilibrium with respect to the loading $L_{j}:=\left(T_{j}, D_{j}\right)$ if the following inequality holds:

$$
c_{1}^{2}(j)<\left|c_{1}\right|_{\text {crit }}^{2}
$$

where $\left|c_{1}\right|_{\text {crit }}^{2}$ is given in (4.8) and $c_{1}(j)=c_{1}\left(\Gamma_{j}, L_{j}\right)$ is the coefficient of the expansion (3.27), computed for the field $u$ at step $j$, corresponding to the crack $\Gamma_{j}$ and loading conditions $L_{j}$.

The passage from step $j$ to $j+1$, for $j<N$, is computed as follows:

Algorithm Assume that $\Gamma_{j}$ is in equilibrium with respect to the loading $L_{j}$. Then:

(1) Change the loading to $L=\left(T_{j+1}, D_{j+1}\right)$, keeping the crack configuration $\Gamma=\Gamma_{j}$ fixed.

(2) Compute $c_{1}(\Gamma, L)$.

a): If $c_{1}^{2}(\Gamma, L)<\left|c_{1}\right|_{\text {crit }}^{2}$ then we are still in an equilibrium point. If $j+1<N$ put $j=j+1, \Gamma_{j+1}=\Gamma_{j}$ and proceed to step 1) again.

b): If $c_{1}^{2}(\Gamma, L) \geq\left|c_{1}\right|_{\text {crit }}^{2}$, then compute $c_{2}(\Gamma, L)$ (cf. (3.27)). Add to $\Gamma$ a single kink of length $l$ and angle $\varphi=-\frac{4 \pi}{3} \frac{c_{2}}{c_{1}} l^{1 / 2}$ (cf. (4.17)). Renovate $\Gamma$ :

$$
\Gamma=\Gamma \cup \operatorname{kink}(l, \varphi) .
$$

If the moving tip of $\Gamma$ lies outside $\Omega$ stop the propagation. In other case repeat step 2).

Observe that the complete breakdown of the body may be achieved before all the steps of loading are carried out. This is consistent with the physics, as soon as we realize that the physical time scale is very long compared with $\Delta t$. Moreover, we should take care that the excess of energy in step 2,b), given by the difference $c_{1}^{2}(\Gamma, L)-\left|c_{1}\right|_{\text {crit }}^{2}$, is not very large, otherwise the quasistatic propagation assumption is violated. It is easy to add a single step in the algorithm controlling this excess. Notice that the propagation process should be performed in a situation such that $c_{1}^{2}(\Gamma, L) \gtrsim\left|c_{1}\right|_{c r i t}^{2}$. If a stable equilibrium is reached we change the loading keeping the crack fixed. If the driving force is equal or greater than the resistance we create more crack surface until a new equilibrium is attained. The underlying description is common to any quasistatic process in mechanics.

\section{Open questions AND Final Remarks.}

We addressed the study of the path followed by a propagating crack in a quasistatic regime in out-of-plane field. The problem is modelled by means of a discrete version of the maximum energy release rate criterion, keeping a "local" point of view on the optimal direction of propagation and Griffith's growing condition (cf.1.1). We address some open questions and related problems.

The approach is well suited for numerical study. The angle condition of step $2 \mathrm{~b}$ ) in the algorithm is easier to handle than the symmetry principle (cf. [18]). The corresponding numerical method should use an efficient way to compute the coefficients $c_{1}$ and $c_{2}$ in (3.27). This may be achieved by the application of $J$ integrals (cf. [16]).

The well-possedness of the scheme, as well as the analysis of further conditions on the "time" step and the size of the kink ensuring convergence for both parameters tending to zero, are also matter of future research. The regularity of the limiting curve, for $l, \Delta t \rightarrow 0$ is interesting as well. According to our result, at any point 
of the limiting curve where $c_{2} \neq 0$ the curvature of the path turns to be infinite (cf. 4.18). It would be interesting to establish if $c_{2}=0$ is a kind of "continuum limit condition" for the crack path, analogous to the symmetry principle for the mixed modes.

Acknowledgements This research was carried out while the author was visiting the Max Planck Institute for Mathematics in the Sciences during the period octobernovember 2002 and the academic year 2003-2004. The author wishes to express his deep gratitude to Prof. Stephan Luckhaus for enlightening discussions during the preparation of this manuscript as well as to professors Miguel A. Herrero and José Fernández Sáez for valuable comments about the final work.

\section{REFERENCES}

[1] M. Amestoy and J.B. Leblond, Crack paths in plane situations II: Detailed form of the expansions of the stress intensity factors, Int. J. Solids Structures, Vol. 29, Nr. 4 (1992), pp. 465-501.

[2] C.M.Bender and S.A. Orszag, Advanced Mathematical Methods for Scientists and Engineers, International Edition, Mc Graw-Hill Book Co. Singapore (1978).

[3] M. Brokate and A. Khludnev, On crack propagation shapes in elastic bodies, Z. angew. Math. Phys. 55 (2004), pp.318-329.

[4] M. Buliga, Energy minimizing brittle crack propagation, Journal of Elasticity, 52 (1999), pp. 201-238.

[5] M. Buliga, Brittle crack propagation based on an optimal energy balance, Rev. Roum. des Math. Pures et Appl., 45, 2 (2001), pp 201-209.

[6] B. Cotterell and J.R. Rice, Slightly curved or kinked cracks, International Journal of Fracture, Vol. 16, No. 2 (1980), pp. 155-169.

[7] G. Dal Maso and R. Toader, A model for the quasi-static growth of brittle fracture: existence and approximation results, Archive for Rational Mechanics and Analysis, 162, No.2 (2002), pp. 101-135.

[8] G.A. Francfort and C.J. Larsen, "Existence and convergence for quasi-static evolution in brittle fracture", Communications on Pure and Applied Mathematics, Vol. LVI (2003), pp. 14651500 .

[9] G.A. Francfort and J.J. Marigo, Revisiting brittle fracture as an energy minimization problem, Journal of the Mechanics and Physics of Solids, Vol.46, No.8 (1998), pp.1319-1342.

[10] L.B. Freund, Dynamic Fracture Mechanics, 2d edition. Cambridge University Press, Cambridge (1998).

[11] R.V. Goldstein and R.L. Salganik, Brittle fracture of solids with arbitrary cracks, International Journal of Fracture, 10 (1974), pp. 507.

[12] A.A. Griffith, The phenomenon of rupture and flow in solids, Phylosophical Transactions of the Royal Society (London), A 221 (1920), pp. 163-198.

[13] G.R. Irwin, Analysis of stresses and strains near the end of a crack transversing a plate, Journal of Applied Mechanics 24 (1957), pp. 361-364.

[14] J.B.Leblond, Crack paths in plane situations I: General form of the expansion of the stress intensity factors, Int. J. Solids Structures, Vol. 25, nr. 11 (1989), pp. 1311-1325.

[15] Z. Nehari (1975), Conformal Mapping, Dover Publications, New York (1975).

[16] J.R. Rice, Mathematical analysis in the mechanics of fracture. In: H. Liebowitz (ed.) Fracture, An Advanced Treatise, Vol.2, ed. New York: Academic Press (1968), pp.191-311.

[17] G.C.Sih, Stress distribution near internal crack tips for longitudinal shear problems, Journal of Applied Mechanics (march 1965), pp. 51-58.

[18] T.J.Stone and I. Babuska, A numerical method with a posteriori error estimation for determining the path taken by a propagating crack, Computer Methods in applied mechanics and engineering, 160 (1998), pp. 245-271.

[19] Chien H.Wu, Elasticity problems of a slender Z-crack, Journal of Elasticity, Vol.8, No.2 (1978), pp. 235-257. 


\section{Appendix A. The BASIC CONFORMAL MAP FOR Kinked CONFIGURATions}

In order to transform the upper half plane into the plane with a semi infinite slit and a small kink around the origin we will apply a Schwarz-Christoffel-type of transformation. If $\zeta$ is the variable for the kinked configuration and $z$ is the one corresponding to the upper half plane, we look for a conformal map $\zeta=f_{l}(z)$ that satisfies:

$$
d \zeta=f_{l}^{\prime} d z=k(z-a)^{-\varphi / \pi} z(z-b)^{\varphi / \pi} d z \quad a<0<b .
$$

This means that for $z \in \mathbb{R}$ and $d z>0$, when $z$ goes from $a^{-}$to $a^{+}$we have a jump in the argument of $z-a$ of magnitude $-\pi$. Then, the exponent adjusts the jump of $d \zeta$ to the angle $\varphi$. When we cross 0 , we have another change in the argument of $d \zeta$ of amount $-\pi$. As $z$ grows we are going back to zero, in the $\zeta$ plane. We must select the value of $b$ in such a way that the next turn is made in this point, with an angle $\varphi$. In this way, for $z>b, \zeta$ must lie in the real negative axis, with $d \zeta<0$ for $d z>0$. We also have to select the constant $k$ in order to start with a positive $d \zeta$ for $d z>0$, to eventually end with a negative one. The values of $k, a$ and $b$ depend on $l$ and $\varphi$. We compute this parameters in the following paragraphs.

Let us call:

It is easy to check that

$$
\alpha:=\frac{\varphi}{\pi} .
$$

$$
\int^{z}(\zeta-a)^{-\alpha} \zeta(\zeta-b)^{\alpha} d \zeta=\frac{1}{2}(z-a)^{1-\alpha}(z-b)^{1+\alpha},
$$

for real constants $a<b$ satisfying:

$$
\frac{a}{b}=-\frac{(1-\alpha)}{(1+\alpha)}
$$

From (A.1) we obtain the following expression for $f_{l}$ :

$$
f_{l}(z)=\frac{k}{2}(z-a)^{1-\alpha}(z-b)^{1+\alpha}
$$

where we also imposed that $f_{l}(a)=0$. Notice that we have then $f_{l}(b)=0$, ie. the two values $a$ and $b$ correspond to the kink corner located at the origin. The kink tip corresponds to the origin in the $z$ plane, ie. we must have that $f_{l}(0)=l e^{i \varphi}$.

We now proceed to compute constants $a, b$ for a given length of the kink $l$. From (A.3) and (A.2), we should have that:

$$
f_{l}(0) \equiv l e^{i \varphi}=-\frac{k}{2} e^{i \varphi}(-a)^{1-\alpha}(b)^{1+\alpha}
$$

and we obtain:

$$
l=-\frac{k}{2}(-a b)\left(-\frac{b}{a}\right)^{\alpha}
$$

We have then the following two equations for $a$ and $b$ :

$$
\frac{a}{b}=-\frac{1-\alpha}{1+\alpha}, \quad a b=\frac{2 l}{k}\left(\frac{1-\alpha}{1+\alpha}\right)^{\alpha} .
$$

Multiplying both:

$$
a^{2}=-\frac{2 l}{k}\left(\frac{1-\alpha}{1+\alpha}\right)^{1+\alpha} \rightarrow a=-\sqrt{-\frac{2 l}{k}}\left(\frac{1-\alpha}{1+\alpha}\right)^{\frac{1+\alpha}{2}} \quad(a<0) .
$$


And we have then that:

$$
b=\sqrt{-\frac{2 l}{k}}\left(\frac{1+\alpha}{1-\alpha}\right)^{\frac{1-\alpha}{2}} .
$$

For $l=0$, we want to recover the transformation:

$$
f_{0}(z)=-z^{2}
$$

and then we can fix a value for $k$. We have from (A.4) and (A.5) that $a=0$ and $b=0$ for $l=0$. Going back to (A.3) we have that:

$$
f_{0}(z)=\frac{k}{2} z^{2}
$$

and then we obtain (cf. (A.6)):

$$
k=-2 .
$$

We summarize the results in the following formulae:

$$
\begin{aligned}
f_{l}(z) & =-(z-a(l))^{1-\alpha}(z-b(l))^{1+\alpha}, \\
a(l) & =-\sqrt{l}\left(\frac{1-\alpha}{1+\alpha}\right)^{\frac{1+\alpha}{2}}, \\
b(l) & =\sqrt{l}\left(\frac{1+\alpha}{1-\alpha}\right)^{\frac{1-\alpha}{2}} .
\end{aligned}
$$

Departamento de Matemática Aplicada, Facultad de Matemáticas, Universidad ComPlutense de Madrid, 28040 Madrid (Spain)., Max Planck Institute for Mathematics in the Sciences, Leipzig, Germany.

E-mail address: oleaga@mat.ucm.es 